Sitientibus Série Ciências Físicas 06: 1-10 (2010)

\title{
Propagação de Ondas Eletromagnéticas através de um Sistema Multicamadas Aleatórias
}

\author{
Propagation of Electromagnetic Waves through a Random Multilayer System
}

\author{
Antônio Delson C. de Jesusf e Waldeck Sotero Coutinho \\ Departamento de Física - UEFS \\ Campus Universitário, $\mathrm{km}$ 03, BR 116, \\ Feira de Santana - BA - 44036-900
}

\begin{abstract}
Neste trabalho estudamos a resposta de um sistema de multicamadas aleatórias constituído de camadas homogêneas, a um campo eletromagnético no modo TE (Transversal Elétrico) limitando-se à incidência normal. Verificamos que a transmissão das ondas se dá de forma periódica de modo que existem bandas de modos transmissíveis com relação ao número de onda. Daí, isolamos um único espectro de ondas e verificamos a inufluência da quantidade de camadas e da largura de desordem na resposta do sistema. Os resultados mostraram que no limite de grandes quantidades de camadas a média espectral da banda transmissível varia com uma lei de potência. A largura de desordem da média espectral decresce exponencialmente.
\end{abstract}

Palavras-chaves: Transmissividade, Multicamada, Aleatória.

We study the response of a system of random multilayers consisting of homogeneous layers, to an electromagnetic field mode TE (Transverse Electric) when this is limited to the normal incidence. We found that the transmission of the waves takes place in a periodic fashion so that there are bands of modes transmitted with respect to the wavenumber. Hence, we isolate a single wave spectrum and observed the influence of the number of layers and the width of disorder in the system response. The results show that in boundary layers of large quantities of the average spectral band the transmissivity varies with a power law. The width of the average spectrum disorder decreases exponentially.

Key-words: Transmissivity, Multilayer, Random.

\section{INTRODUÇÃO}

Em meados do século passado existia a necessidade de se solucionar o problema da inibição de emissões espontâneas em alguns sistemas físicos [1, 2] tais como o laser, transistores bipolares de hetero-junção e células solares. Este problema é caracterizado pelo fato da radiação devido às emissões espontâneas não cessarem conjuntamente após a excitação no sistema. Em 1986 Yablonovich investigou a possibilidade num sistema de multicamadas binário, composto de dois tipos de camadas dielétricas dispostas periodicamente

*Endereço Eletrônico: a1d1j1@uefs.br com relação ao índice de refração, com espessura e com dimensões comparáveis ao comprimento da onda incidente. Desta forma, fora possível inibir as radiações espontâneas não desejáveis. Ele identificou a banda proibida no sistema periódico e introduziu o bandgap fotônico [2], chamando assim o sistema de cristal fotônico em analogia aos cristais atômicos, em arranjos uni, bi e tridimensionais [3]. No estudo destes sistemas de multicamadas dielétricas, pesquisadores têm estudado a reflexão total sem absorção, filtragens, etc..

Em 1987 Kohmoto demonstrou a localização da luz, usando um sistema composto por um conjunto de camadas dispostas de acordo com uma seqüência de Fibonacci [4, 5]. Nascimento e outros [6, 7] estudaram o caso binário com amostras arranjadas aleatoriamente e es- 
tabeleceram uma lei de escala. Neste trabalho, estudamos um sistema de multicamadas de alto grau de aleatoriedade, diferentemente dos casos anteriores. A amostra é composta de diversas camadas e, para cada camada, sorteamos um índice de refração diferente, calculado a partir de valores aleatórios da permissividade elétrica e de valores constantes e iguais a 1 da permeabilidade magnética. Tal inferência é compatível com as radiações eletromagnéticas incidentes da região do visível em materiais transparentes, uma vez que para estas respectivas regiões tais materiais são muito pouco magnéticos [8], o que nos possibilita fazer aquela aproximação. As nossas simulações consideram a região do espectro visível e outras regiões de interesse.

\section{FUNDAMENTAÇÃO TEÓRICA E FORMULAÇÃO MATEMÁTICA}

No modelo estudado consideramos inicialmente o caso mais geral possível de desordem, tomando as camadas com permissividade elétrica e permeabilidade magnética variáveis e com as ondas eletromagnéticas incidindo obliquamente sobre a face da primeira camada da amostra de filmes de multicamadas. Nas simulações numéricas, entretanto, consideramos cada camada da amostra possuindo permissividade diferente das demais e frequência da onda constante. Com esta abordagem, queremos modelar amostras dopadas por impurezas doadoras ou mesmo amostras que trazem impurezas naturais que interfiram em suas propriedades elétricas. A permeabilidade magnética é considerada constante em toda a amostra. Esta estrutura é considerada de alto grau de desordem, ou numa generalização do caso aleatório. O efeito da desordem sobre a função de onda é previsto pelo modelo de Anderson [2, 6, 9] desenvolvido para o caso eletrônico, tomado como analogia neste estudo. Para conhecer os campos em qualquer das interfaces (uma vez que no interior eles são nulos), fizemos uso do Método da Matriz de Transferência. Em todos estes casos, conside- ramos a validade da lei de Bragg, como veremos.

\section{A. Método da Matriz de Transferência}

Através deste método, podemos saber qual o campo resultante na última camada de uma amostra constituída de várias camadas arranjadas unidimensionalmente, após uma incidência normal de um campo eletromagnético na primeira camada, onde a direção de propagação coincide com a de $z$. Trata-se de um sistema de multicamadas dielétricas arranjadas unidimensionalmente. Consideremos um campo elétrico perpendicular ao plano de incidência, polarizado no modo TE. Para um meio sem cargas livres e densidade de corrente, as equações de Maxwell não se alteram quando se faz permutas simultâneas do campo elétrico, $\overrightarrow{\mathrm{E}}$, com a intensidade magnética, $\overrightarrow{\mathrm{H}}$, e da permissividade elétrica $\epsilon$ com o negativo da permeabilidade magnética, $-\mu$. Daí, consideramos apenas o modo $T E$ e não o $T M$ (campo magnético polarizado perpendicular ao plano de incidência). Se a polarização for arbitrária podemos separar a onda em duas, uma do tipo $T E$ e outra do tipo $T M$ e estudá-las separadamente, uma vez que as condições de contorno nas interfaces são independentes. Então, escolhemos a onda no modo $T E$ e o plano de incidência como sendo $Y Z$. Neste caso, a polarização fica na direção de $x \mathrm{e}$, portanto, $\mathrm{E}_{y}=0$ e $\mathrm{E}_{z}=0$. Assumindo uma dependência temporal do tipo exponencial $\mathrm{e}^{(-i \omega t)}$, os campos adquirem as seguintes formas:

$$
\begin{aligned}
\overrightarrow{\mathrm{E}}(x, y, z)= & \mathrm{E}_{x}(x, y, z) \hat{\imath}+0 \hat{\jmath}+0 \hat{k}, \\
\overrightarrow{\mathrm{H}}(x, y, z)= & \mathrm{H}_{x}(x, y, z) \hat{\imath}+\mathrm{H}_{y}(x, y, z) \hat{\jmath} \\
& +\mathrm{H}_{z}(x, y, z) \hat{k} .
\end{aligned}
$$

Para estes campos as equações de Maxwell são resolvidas no sistema gaussiano em meio dielétrico, na direção de estratificação $z$, considerando-se também as relações constitutivas para os campos $(\overrightarrow{\mathrm{D}}=\epsilon \overrightarrow{\mathrm{E}})$ e $(\overrightarrow{\mathrm{H}}=1 / \mu \overrightarrow{\mathrm{B}})$. 
Assim, obtemos,

$$
\begin{aligned}
& \frac{d^{2} U(z)}{d z^{2}}-\frac{d[\ln \mu(z)]}{d z} \frac{d U(z)}{d z} \\
& +k_{0}^{2}\left(\eta^{2}-\alpha^{2}\right) U(z)=0,
\end{aligned}
$$

como também,

$$
\begin{aligned}
& \frac{d^{2} V(z)}{d z^{2}}-\frac{d}{d z}\left[\ln \left(\epsilon-\frac{\alpha^{2}}{\mu}\right)\right] \frac{d V(z)}{d z} \\
& +k_{0}^{2}\left(\eta^{2}-\alpha^{2}\right) V(z)=0,
\end{aligned}
$$

onde $n\left(n^{2}=\epsilon \mu\right)$ é o índice de refração, $k_{0}$ $\left(k_{0}=\omega / c=2 \pi / \lambda_{0}\right)$ é o número de onda, $\omega$ é a frequência angular da onda, $c$ é a velocidade da luz no vácuo e $\lambda_{0}$ é o caminho ótico. Nestas equações $U(z)$ é a amplitude do campo elétrico na direção $x, V(z)$ e $W(z)$ são as amplitudes da intensidade magnética nas direções $y$ e $z$, respectivamente e $K=k_{0} \alpha$. O sistema de equações acima representa a propagação das ondas eletromagnéticas incidentes na amostra heterogênea. Assumindo o modelo de onda plana, existem superfícies tal que a amplitude do campo pode ser considerada constante e superfícies em que a fase também é constante. Neste caso, tomemos as equações que dão as superfícies de amplitude e de fase constantes de $\mathrm{E}_{x}$, tal que, em cada plano que corta cada ponto do eixo $z$ perpendicularmente, os campos atingem mesmos valores de campo máximo e existem conjuntos de pontos $(y, z)$ formando superfícies planas, tal que as fases correspondentes a estes são constantes.

Seja $\varphi(z)$ uma possível fase da amplitude $U(z)$. A lei de Snell para um meio estratificado com ângulo de incidência $\theta$ é,

$$
\varphi(z)=k_{0} \eta z \cos \theta
$$

Para as nossas simulações, adotamos permeabilidade constante e igual a 1 e permissividade aleatória em cada camada da amostra. Sendo assim, as equações diferenciais são simplificadas,

$$
\begin{aligned}
& \frac{d^{2} U(z)}{d z^{2}}+\left(k_{0}^{2} \eta^{2} \cos ^{2} \theta\right) U(z)=0 \\
& \frac{d^{2} V(z)}{d z^{2}}+\left(k_{0}^{2} \eta^{2} \cos ^{2} \theta\right) V(z)=0 .
\end{aligned}
$$

Considerando condições de contorno apropriadas, uma solução particular das equações diferenciais, $[U(z), V(z)]$, é encontrada e, assim, definimos a matriz característica de uma camada dielétrica homogênea qualquer, sujeita a um campo eletromagnético no modo $T E$ incidindo obliquamente. Para as características do nosso modelo, a matriz $M$ é dada por,

$$
\begin{aligned}
& M(z)= \\
& {\left[\begin{array}{cc}
\cos \left(k_{0} \eta z \cos \theta\right) & i p \operatorname{sen}\left(k_{0} \eta z \cos \theta\right) \\
-i p \operatorname{sen}\left(k_{0} \eta z \cos \theta\right) & \cos \left(k_{0} \eta z \cos \theta\right)
\end{array}\right],}
\end{aligned}
$$

onde,

$$
p=\sqrt{\frac{\epsilon}{\mu}} \cos \theta
$$

Esta matriz $M$ advinda das equações de Maxwell é geral, considerando a permeabilidade magnética variando com $z$. Ela é chamada matriz característica de um meio estratificado. Se $Q_{0}$ for a matriz das amplitudes do campo eletromagnético incidente e $Q_{N}$, a matriz da resposta a este campo, a resposta ao campo incidente na primeira camada pode ser encontrada através da matriz $M$, tal que:

$$
Q_{0}=M_{1} \cdot M_{2} \cdot M_{3} \cdots M_{N} Q_{N} .
$$

Para restringirmos estes resultados aos de um sistema com incidência normal (o que é o caso que tratamos aqui), basta fazer $\theta=0$ graus, diretamente na equação. Para esta incidência e tomando $z$ como sendo a largura da camada correspondente e fizermos $k_{0} \eta z=m \pi, m$ inteiro, onde $\eta z=\lambda_{0}$ e, portanto, $k_{0} \lambda_{0}=m \pi$, a matriz se torna uma identidade, o que implica em um campo com transmissividade máxima para estas radiações com estes números de onda correspondentes, de forma que a variação da transmissividade é periódica. Como estas transmissividades ocorrem para $k_{0}=m \pi / \lambda_{0}$, e uma vez que o número de onda das radiações é dado por $k=2 \pi / \lambda$, temos $k=k_{0}$ e $\lambda=2 \lambda_{0} / \mathrm{m}$. Contudo, é suficiente considerarmos apenas um espectro, ou seja, $m=1$. 


\section{B. Transmissividade e Reflexividade}

O modelo de trasmissividade e reflexividade que adotamos pode ser visto na Figura 1. Consideramos uma onda plana no modo $T E$ incidindo numa estrutura empilhada de camadas com índices de refração aleatórios, cuja direção de estratificação é a de $z$, assim como a de propagação da onda. Neste caso, dispomos a estrutura de modo a ficar com a face externa incidente da primeira camada em $z=0$ e a face externa, de resposta da radiação, na última em um $z=z_{l}$.

Para obtermos os coeficientes de transmissão e de reflexão, consideramos $A$ e $R$ as amplitudes dos campos elétricos incidentes e refletidos, respectivamente, em $z=0$, e $T$ a do transmitido pela superfície em $z=z_{l}$. As ondas transmitidas por qualquer interface serão a onda incidente do meio dielétrico na interface seguinte. Depois de escrevermos o campo magnético em função do campo elétrico para este sistema, obtemos a relação entre as amplitudes dos campos para este meio (com componente magnética nula na direção $z$, na camada dielétrica homogênea),

$$
V_{l}=p_{l} U_{l}=p_{l} T
$$

Aplicando as condições de contorno para os campos elétrico e magnético nas interfaces, os coeficientes de transmissão e reflexão são dados por:

$t=\frac{T}{A}=\frac{2 p_{1}}{\left[\left(m_{11}+m_{12} p_{l}\right) p_{1}+\left(m_{21}+m_{22} p_{l}\right)\right]}$,

$r=\frac{R}{A}=\frac{\left(m_{11}+m_{12} p_{l}\right) p_{1}-\left(m_{21}+m_{22} p_{l}\right)}{\left[\left(m_{11}+m_{12} p_{l}\right) p_{1}+\left(m_{21}+m_{22} p_{l}\right)\right]}$.

$\mathrm{O}$ índice 1 indica a primeira camada e através das Equações (12) e (13) podemos obter a transmissividade e a reflexividade com as densidades de energias médias.

\section{RESULTADOS}

Simulamos numericamente sistemas de multicamadas com alto grau de aleatoriedade, atribuindo para os valores da permissividade uma distribuição de probabilidade de números aleatórios. Consideramos as amostras com esta característica como sendo heterogêneas. Às amostras com permissividade constante denominamos homogêneas. Para qualquer caso, consideramos a permeabilidade do vácuo constante e igual a 1.

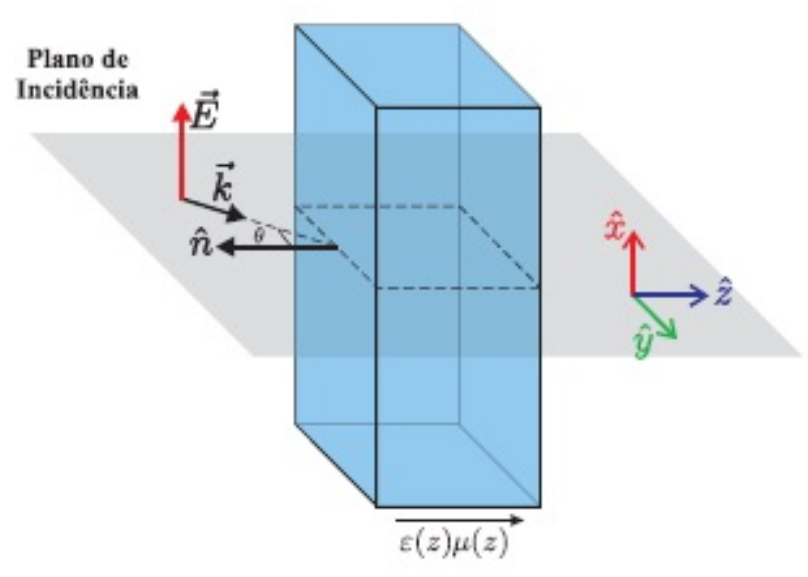

FIGURA 1: Propagação da onda em uma estrutura de multicamadas. Fonte: Nascimento [6].

Os resultados mostram como a transmissividade média e a média espectral variam em função do número de onda, da largura de desordem, número de camadas da amostra. Além disso, consideramos para esta análise larguras de desordem para índices de refração médios dentro de intervalos de validade dos fenômenos físicos. O estudo dos resultados da média espectral foi realizado no sentido de se obter expoentes característicos dos modos transmissíveis e não transmissíveis da radiação.

\section{A. Transmissividade Média vs Número de Onda}


Os resultados mostram que a transmissividade média decresce com o aumento do número de onda, como pode ser visto na Figura 2. Esta transmissividade média é a resultante das radiações por cada amostra. Usamos nesta fase amostras homogêneas e heterogêneas e comparamos seus resultados. As simulações foram realizadas para 12 amostras, 100 camadas e caminho ótico igual a $5 \times 10^{-6}$. Os resultados mostraram que a amplitude da transmissividade decresce com o número de onda para qualquer caso (homogêneo e heterogêneo).

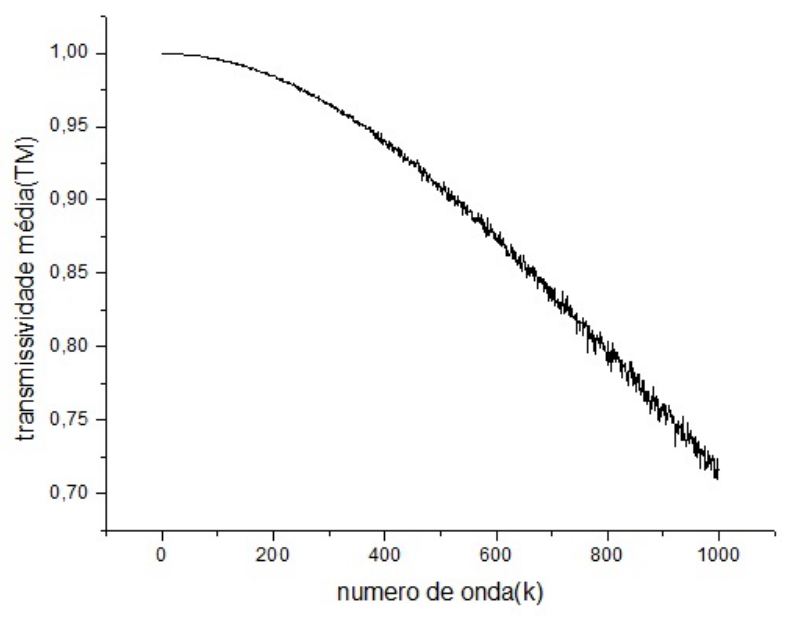

FIGURA 2: Transmissividade média em função do número de onda, caso heterogêneo.

O caso heterogêneo (Figura 2) mostra ruído em relação ao homogêneo, que identifica valores aleatórios para a permissividade em cada camada diferente. Além disso, observamos que para números de onda maiores, aparece a periodicidade da transmissividade média com o número de onda.

Este fato pode ser verificado na Figura 3 (caso homogêneo), na qual o número de onda foi aumentado 100 vezes em relação ao valor inicial. Dos resultados podemos verificar a periodicidade numa variação tipo senoidal entre, aproximadamente, os valores de transmissividade 0,8 e 1,0 , com período em torno de $k=6.300$, onde ocorrem valores máximos

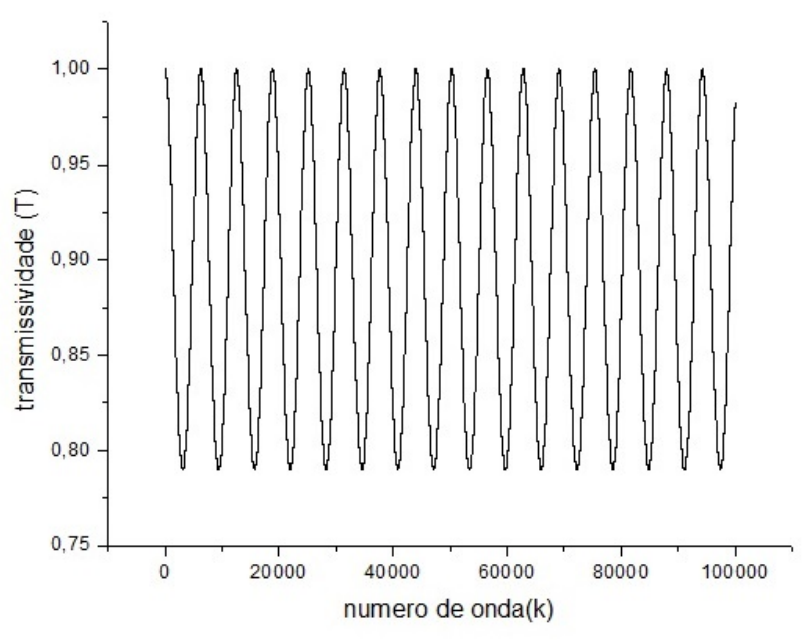

FIGURA 3: Transmissividade em função do número de onda (até 105), caso homogêneo.

para múltiplos inteiros deste e mínimos para seus múltiplos semi-inteiros (Figura 3). Este mesmo fenômeno ocorre para o caso heterogêneo, contudo com a amplitude da transmissividade média decrescendo exponencialmente em relação ao caso homogêneo. Claramente vemos uma dependência da transmissividade média com o número de onda.

\section{B. Transmissividade Média vs Número de Camadas}

Verificaremos adiante se a transmissividade varia com o número de camadas da amostra, no sentido de investigarmos se ela é atenuada ou não ao longo das camadas. Na Figuras 4. abaixo, apresentamos o comportamento para 1000 camadas, 12 amostras, número de onda igual a 8.000 e caminho ótico igual a $5 \times 10^{-6}$ para o caso heterogêneo.

Claramente, observamos uma atenuação da onda ao longo do número de camadas. O caso homogêneo foi simulado para 10.000 camadas e não apresentou atenuação, como esperado. 


\section{Transmissividade Média e Permissividade Relativa (Meio Homogêneo)}

Nesta seção apresentamos os resultados para as simulações de amostras homogêneas, cada uma caracterizada por uma permissividade relativa diferente. Ou seja, para cada amostra composta de diversas camadas, cada camada possui um mesmo valor da permissividade e esta permissividade varia de amostra para amostra. O objetivo é ver se o comportamento da transmissividade varia de amostra para a mostra. Também consideramos valores de número de onda diferentes, a fim de ver se é mantido um mesmo comportamento. $\mathrm{O}$ gráfico da Figura 5, a seguir, mostra este resultado para o caminho ótico $\lambda_{0}=5 \times 10^{-6}$ e 100 camadas. As simulações mostraram que o aumento no número de onda caracteriza com mais clareza o comportamento da transmissividade em relação à permissividade relativa. Observamos que a amplitude da transmissividade decresce exponencialmente em relação à permissividade relativa para números de onda altos e tem comportamento linear quando eles são pequenos. O gráfico da Figura 5 mostra o comportamento geral do fenômeno.

\section{Transmissividade Média e Largura de Desordem}

Nesta etapa da investigação, buscamos verificar o que ocorre com o espectro da transmissividade média em função do número de onda ao variarmos a largura de desordem. A largura de desordem corresponde ao tamanho do intervalo de valores reais, incluindo positivos e negativos, geralmente eqüisseparados, a serem somados com um índice de refração, extraindo-se daí um valor médio para o índice de refração. Estes valores de dentro da faixa da largura podem ser aleatórios ou não. Deste modo os índices de refração das camadas estão determinados dentro das faixas, dadas por,

$$
\langle n\rangle-\frac{l d}{2}<n<\langle n\rangle+\frac{l d}{2},
$$

onde, $l d$ é a largura de desordem e $\langle n\rangle$ é o índice de refração médio. Consideraremos uma faixa de valores reais naturais de índices de refração entre 1 e 8,944, dentro da qual escolheremos os índices de refração para cada camada da amostra, cuja flutuação (densidade) é determinada pela largura de desordem.

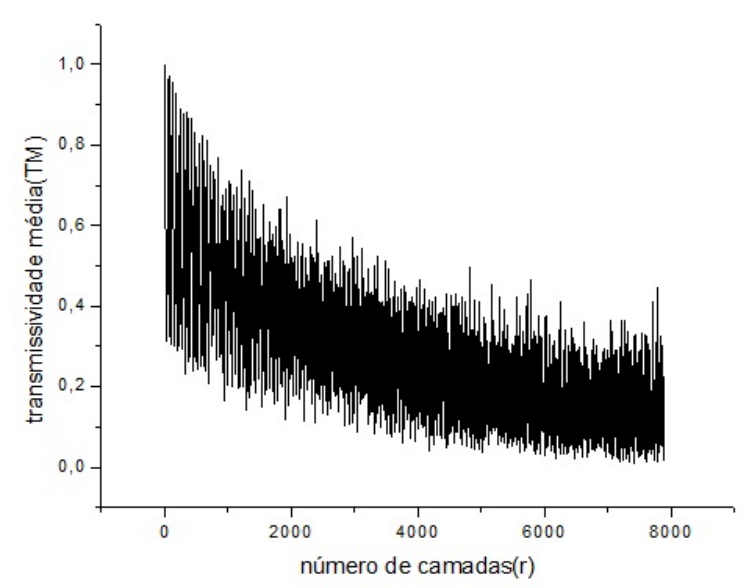

FIGURA 4: Transmissividade em função do número de onda (até 105), caso homogêneo.

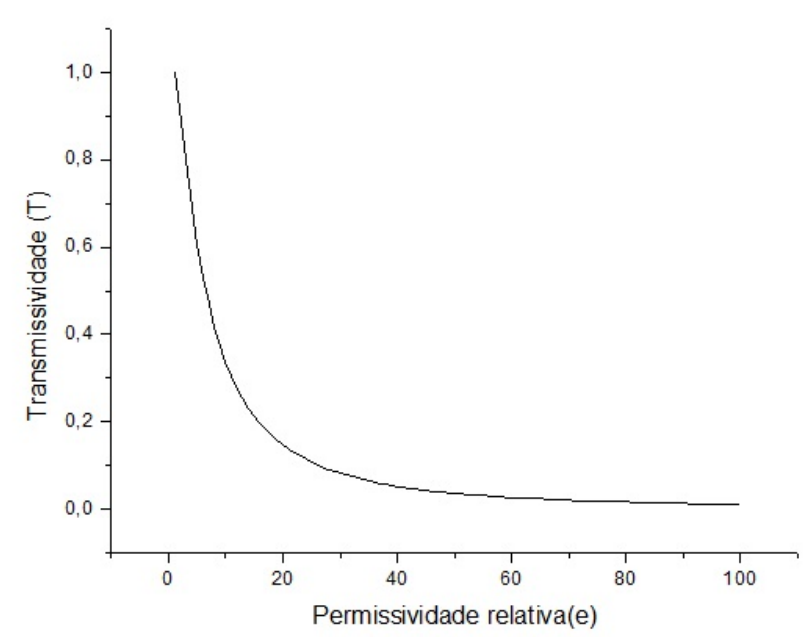

FIGURA 5: Transmissividade em função do número de onda (até 105), caso homogêneo.

Continuamos a comparar meios homogêneos e heterogêneos em que os índices de refração 
dos meios homogêneos, $n$, são iguais aos índices de refração médio $\langle n\rangle$ do meio heterogêneo de modo que obtivemos resultados para índices de refração médios iguais: 1) $\langle n\rangle=1,5,2)$ $\langle n\rangle=4,028$, e 3$)\langle n\rangle=7,056$. As simulações para o caso homogêneo não exibiram nenhuma modificação na amplitude da transmissividade para amostras com índices de refração diferentes. Contudo, ao introduzirmos a largura de desordem, variando o índice de refração no caso heterogêneo, observamos que à medida que aumentamos a largura de desordem, ocorre uma queda da amplitude da transmissividade e um comportamento oscilatório é verificado em relação ao número de onda. Aumentar a largura de desordem equivale a aumentar a distribuição de camadas na amostra em relação ao índice de refração. Além disso, o comportamento oscilatório verificado é justificado pelas interferências destrutivas e construtivas que ocorrem nas interfaces entre as camadas, devido às múltiplas reflexões e transmissões no sistema em estudo. $\mathrm{O}$ aumento no índice de refração exacerba este efeito. A Figura 6 mostra o resultado para o índice de refração médio igual a 1,5 para uma largura de desordem igual a 0,4 .

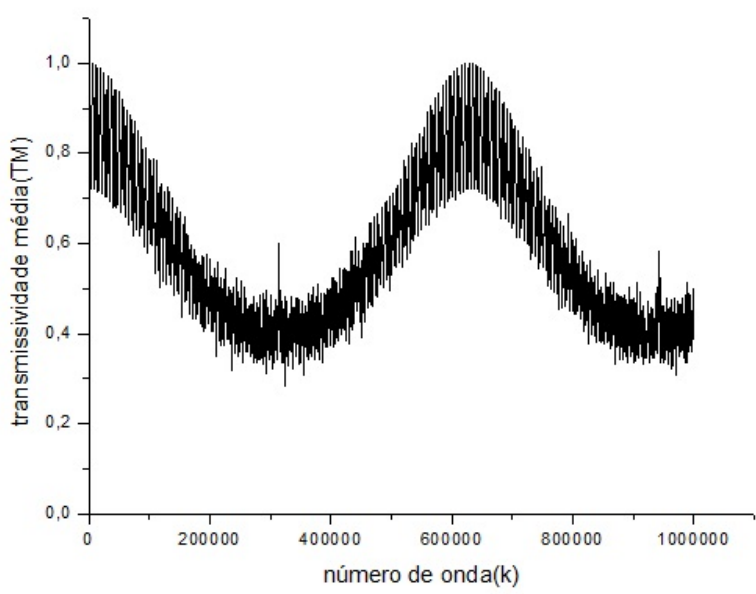

FIGURA 6: Transmissividade em função do número de onda (até 105), caso homogêneo.
O gráfico na Figura 6 mostra uma tendência a uma há seleção dos modos transmissíveis (na formação dos picos). Este fenômeno se confirmará adiante com o aumento da largura de desordem para determinadas faixas dos índices de refração médios. A seguir, nos gráficos das Figuras 7 e 8, a largura de desordem foi aumentada em 5 vezes em relação ao caso mostrado na Figura 6. O índice de refração médio é aumentado na Figura 8 e o fenômeno da seleção dos modos transmissíveis é confirmado.

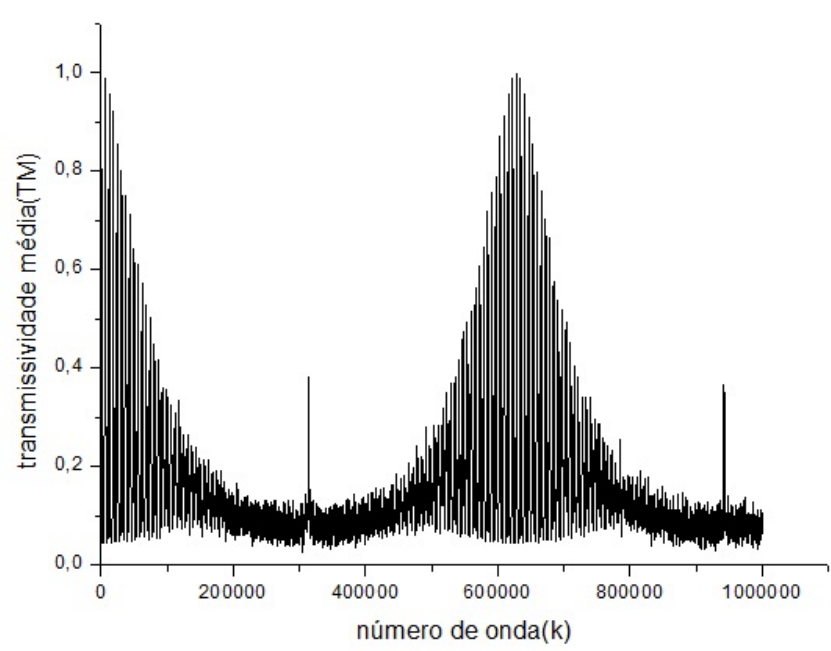

FIGURA 7: Transmissividade em função do número de onda (até 105), caso homogêneo.

Claramente pode ser visto que o aumento da largura de desordem para o índice de refração intermediário $(4,028)$, proporciona uma seleção de modos de transmissão (os picos nas Figuras). Estes modos passam a ficar mais evidentes dentro de determinadas larguras de desordem. Tomando este índice de refração (intermediário) e uma largura de desordem muito pequena, o efeito da desordem é imperceptível, pois uma largura de desordem pequena significa pequenas diferenças entre os valores dos índices de refração que compõem a amostra. Com isto as intensidades de ondas transmitidas na amostra heterogênea não variam muito 


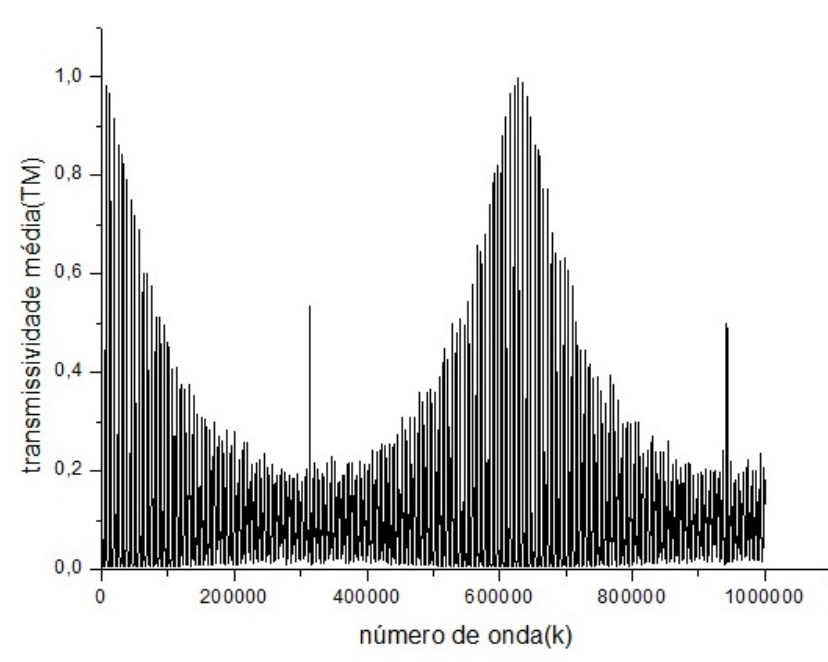

FIGURA 8: Transmissividade em função do número de onda (até 105), caso homogêneo.

(similar a um meio quase homogêneo) de camada a camada. À medida que aumentamos esta largura percebemos os efeitos da distribuição aleatória das diferentes camadas da amostra.

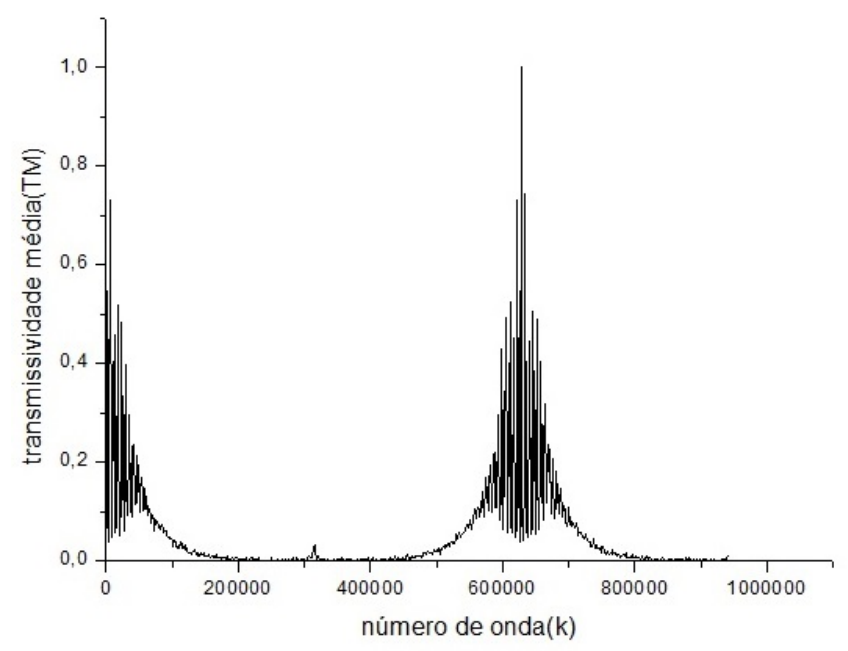

FIGURA 9: Transmissividade em função do número de onda (até 105), caso homogêneo.

Com o gráfico da Figura 9 percebemos que há seleção dos modos transmissíveis à medida que a largura de desordem aumenta para determinadas faixas dos índices de refração médios.
Resultados mais precisos foram obtidos quando aumentamos o número de amostras 5 vezes, e a largura de desordem 2,5 vezes em relação aos casos anteriores (Figuras 7 e 8). Para este caso, escolhemos o índice de refração médio igual a 4,0 , em torno do qual variam aqueles tomados aleatoriamente.

A Figura 9 confirma a seleção dos modos transmissíveis com maior precisão. Os nossos resultados mostram um espectro que apresenta uma seqüência de bandas de modos transmissíveis centradas em frequências, cujos comprimentos de onda no vácuo (ondas incidentes na amostra) associados correspondem à razão do dobro do caminho ótico por números inteiros. Em relação aos resultados da amostra periódica binária (cristal fotônico) obtidos por Nascimento et all (2007), tivemos também a largura da banda de transmissão reduzida e conseqüentemente, a do bandgap fotônico aumentada, porém nesta região surgem alguns modos transmissíveis. Nas proximidades do modo central correspondente ao dobro do caminho ótico, a média espectral diminui com a desordem. Em contrapartida, nas proximidades do modo central correspondente ao quádruplo do caminho ótico, a média espectral aumenta com a desordem.

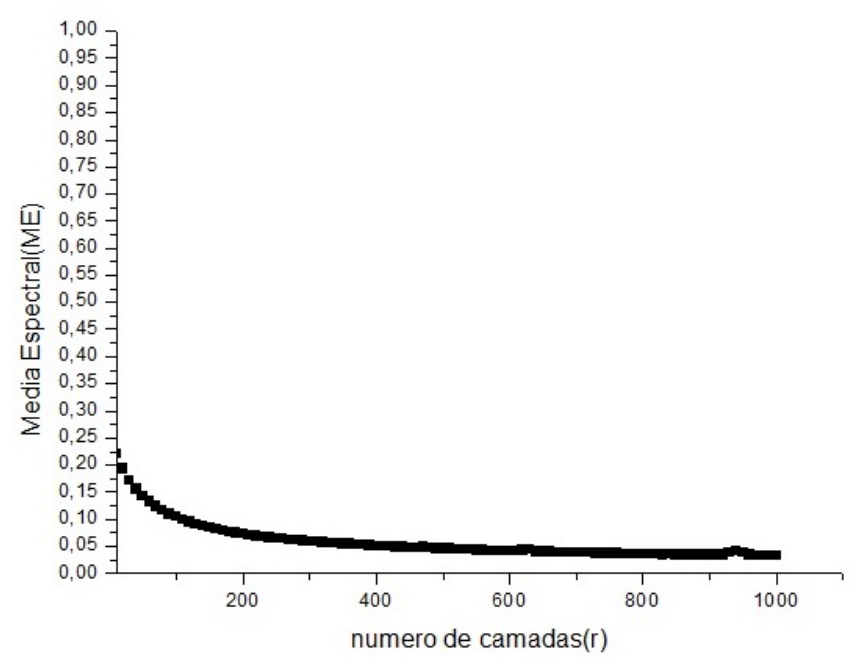

FIGURA 10: Transmissividade em função do número de onda (até 105), caso homogêneo. 
Os gráficos das Figuras 10 e 11 mostram a média espectral (calculada pela média aritmética simples, com os resultados do experimento numérico associado ao problema estudado), ME, e analisamos a sua variação em função da quantidade de camadas na amostra e da largura de desordem, respectivamente.

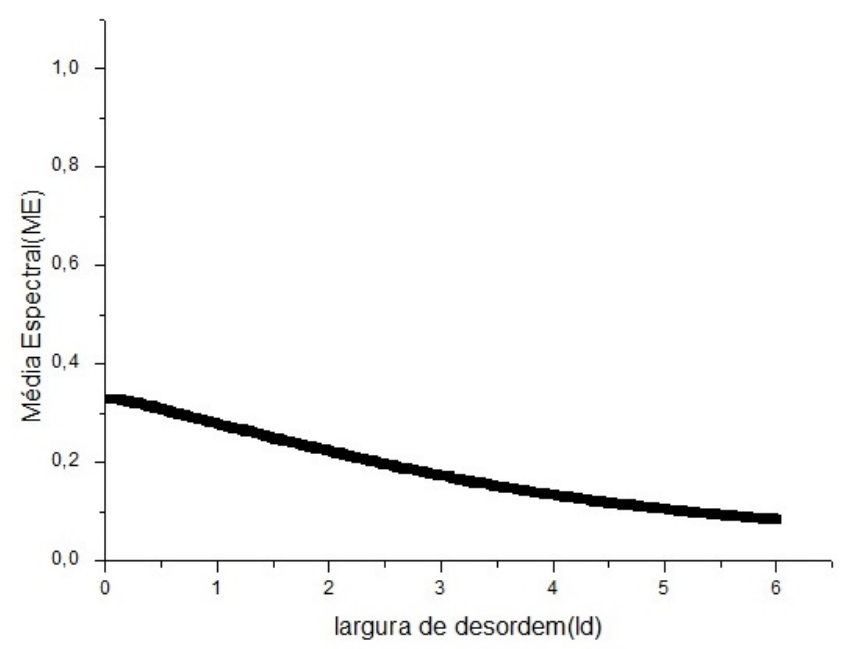

FIGURA 11: Transmissividade em função do número de onda (até 105), caso homogêneo.

Buscamos a caracterização de modos transmissíveis, estudando as respostas de um sistema de multicamadas unidimensional aleatório generalizado a um campo eletromagnético incidido perpendicularmente nas faces das camadas que compõem a amostra. O índice de refração médio é 4,0. Na Figura 10 apresentamos a ME tomada para uma amostra de 100 pontos. Um expoente característico foi encontrado e igual a $-0,50$, quando retiramos os primeiros 9 pontos para evitarmos efeitos de contorno. Este valor concorda com o de Nascimento et all (2007) para o caso binário. Sem a retirada de pontos, o expoente é igual a $-0,47$. Os desvios encontrados são da ordem de $2 \%$ para quantidades de camadas grandes, a partir de 100. A ME tende a zero para um número de camadas muito grande, o que concorda com o fenômeno, visto que existem perdas intensas por reflexão. Assim, verificamos que a retirada de pontos iniciais ou finais da amostra modifica o expoente característico. Além disso, se o número de amostras não for grande suficiente, aparecem picos mesmo no gráfico linearizado, os quais desaparecem quando o número de amostra é aumentado. Isto caracteriza flutuações estatísticas apenas. Os resultados observados para 1000 amostras e 1000 pontos mostram que os expoentes são praticamente iguais àqueles obtidos quando os picos foram retirados. De uma forma geral, observamos que os expoentes encontrados podem variar de acordo o número de pontos e picos que considerarmos no cálculo da ME, particularmente, no processo de linearização do gráfico encontrado.

Neste caso, observamos que se a largura de desordem for muito pequena (o que significa que a diferença entre o valor dos índices de refração das camadas que compõem a amostra é desprezível) as respostas serão aproximadamente como a de um meio homogêneo $(0,436)$. Se a largura de desordem for muito grande, a ME tende ao valor 0,08314 que é muito pequeno. Este fato é explicado pelas perdas variadas por reflexão. Nesta análise dos modos transmissíveis em função da largura de desordem usamos 500 amostras.

\section{CONCLUSÕES}

Os nossos resultados mostraram que incidindo normalmente um campo eletromagnético no modo $T E$ em um sistema de multicamadas com alto grau de desordem, ocorrem modos transmissíveis caracterizados por expoentes num comportamento de lei de potência para a Média Espectral da banda destes modos e que isto é uma função da quantidade de camadas. Além disso, ao variamos a largura de desordem verificamos que esta média tem um comportamento exponencial em relação a banda de modos transmissíveis, de modo que para largura de desordem muito grande esta média tende a um valor pequeno. Desta forma, uma vez que obtivemos uma transmissividade periódica em relação ao número de onda, onde bandas de uma faixa de onda são selecionadas para serem transmitidas 
e outras não transmitidas, após a incidência de uma mistura de ondas, o sistema tratado pode ser de aplicação na Optoeletrônica como um filtro passa-bandas em que só passam as bandas de frequências que desejarmos, tomando como analogia à Eletrônica. Podemos fazer o controle das faixas passantes ou não passantes pelo ajuste dos parâmetros relacionados (número de camadas, largura de desordem). A faixa de ondas é determinada pelo caminho ótico.
[1] E. Yablonovitch, Inibited Spontaneous Emission in Solid-State Physics and Electronics. Phys. Rev. Lett. 58, (20) 2059 (1986).

[2] A.T. de Albuquerque Silva, Antenas Planares Reconfiguráveis do tipo $V$-Estático e tipo Defasamento de Arranjo por Matrizes PBG para Aplicações em Telefonia Móvel Celular. Dissertação (Mestrado) - Instituto de Física da Unicamp, Campinas (2002).

[3] C.R. Silva, Oscilações de Rabi em Redes Óticas Não-Lineares. Dissertação (Mestrado) - Instituto de Física da UFAL, Maceió (2008).

[4] M. Kohmoto, B. Sutherland, K. Iguchi, Localization in Optics: Quasiperiodic Media. Phys. Rev. Lett. 58, 2436 (1987).

[5] G. Taylor, M. Kohmoto, B. Sutherland, P.C. Taylor, Localization Light Waves in Fibonacci Dielectric Multilayers. Phys. Rev. Lett. 72, (5) 633 (1994).

[6] E.M. Nascimento, Aspectos da Transmissão
Eletromagnética em Filmes de Multicamadas. Dissertação (Mestrado) - Instituto de Física da UFAL, Maceió (2009).

[7] E.M. Nascimento, F.A.B.F. Moura, M.L. Lyra, Scaling Laws for the Transmission of Random Binary Dielectric Multilayered Structures. Phys. Rev. B 76, 115 (2007).

[8] E. Hecht, Óptica ( $2^{a}$ Ed.). Lisboa: Fundação Calouste Gulbekian (2002).

[9] M.P. da Silva Júnior, Percolação Quântica em Cadeias Diluídas com Acoplamentos de LongoAlcance. Dissertação (Mestrado) - Instituto de Física da UFAL, Maceió (2008).

[10] S.S. Albuquerque, Violação da Teoria de Escala em Sistemas Unidimensionais com Desordem Diluída e Sistemas com Acoplamentos de Longo Alcance. Dissertação (Mestrado) - Instituto de Física da UFAL, Maceió (2006). 